\title{
Cell phone radiation, pregnancy and sperm: What you don't know, what you need to find out, and what you can do now?
}

\section{Devra Davis}

President of Envorimental Health Trust, USA

ARTICLE INFO

\section{Correspondence to:}

Devra Davis

President of Envorimental Health Trust, USA

e-mail: ddavis@ehtrust.org

\section{Keywords:}

Electromagnetic field

Pregnancy

Reproductive system

Sperm formation

\section{ABSTRACT}

Studies in animals and humans confirm that electromagnetic field (EMF) and radio frequency (RF) affect reproductive health of both males and females. We review recent experimental studies, clinical observations, and some epidemiological reports showing that timing of exposure can be more important than classical dose in affecting outcome. Sperm formation, motility, morbidity, morphology and mitochondrial DNA are affected by RF exposures. Pregnancy outcomes vary with prenatal exposures to cell phone radiation in several animal species. Based on these findings, a number of governments have adopted precautionary policies that protect right of men and women to have healthy children if and when they chose to do so. We review recent science underlying these recommendations and clarify ongoing policy challenges created by the rapidly diffusing technology that has not been adequately evaluated. It is important that adequate funding be provided to train scientists and physicians in the study of bioelectromagnetics, that monitoring systems be set up to evaluate the fast moving adoption of technology, and that precautionary policies be adopted to reduce the potential for long term damage to arise.

J.Exp.Clin.Med., 2013; 30: 267 